\title{
Temporal Variations of Dietary Habits in a High-Risk Area for Upper Gastrointestinal Cancers: a Population-Based Study from Northern Iran
}

\author{
Faezeh Salamat ${ }^{1}$, Shahryar Semnani ${ }^{1}$, Maryam Aboomardani ${ }^{2}$, Gholamreza \\ Roshandel $^{1 *}$
}

\begin{abstract}
Background: Nutrition transition is a global health problem, especially in developing countries. It is known as an important factor for development of different types of health conditions including cancers. Objectives: We aimed to assess the pattern of nutrition transition in a high-risk area for upper gastrointestinal cancers in Northern Iran during the last decade. Materials and Methods: This cross-sectional study was conducted on households of Golestan province, Iran. Data on household food consumption between 2001 and 2010 were obtained from the Statistical Center of Iran. The proportions of households with medium/high consumption of main foods were calculated for each year. Joint point software was used for assessing trends. Annual percent changes (APCs) and 95\% CIs were calculated. Results: In total, 12,060 households were recruited. The APCs (95\% CI) of the proportion of households medium/high consumption of cereals, vegetables, legumes, fish, dairy products and meats were $-3.1(-4.1$ to -2.2$),-2.9(-3.8$ to -2.1$),-2.3(-3.2$ to -1.4$),-2.8(-3.3$ to -2.4$),-1.9(-3.0$ to $-0.9)$ and 2.7 (1.2 to 4.3), respectively. Conclusions: We found significant increase in meat consumption among our population between 2001 and 2010. Our results also suggested significant decreasing trend in consumption of so-called healthy foods including, plant foods, fish, and dairy products. Regarding its correlation with health conditions including cancers, nutrition transition should be considered as a priority in health policy making in our region as well as other high-risk populations. It is recommended to conduct community level interventions to increase consumption of plant foods, fish, and dairy products.
\end{abstract}

Keywords: Temporal variation - dietary habits - nutrition transition - cancer - Iran

Asian Pac J Cancer Prev, 16 (6), 2537-2542

\section{Introduction}

Over the last decade, the rapid developments in the number of related scientific fields and in particular, extent of population-based epidemiological evidence, has supported to clarify the role of diet in prevention and control of morbidity and immature mortality due to non-communicable diseases (NCDs) (World Health Organization, 2003).

Nutritional transition is a global event that means changes in the quantity and quality of dietary patterns (Drewnowski and Popkin, 1997). Economic factors have important effect on diet, nutrition and health of people in a society. When populations become urban and incomes increase, societies may enter different steps that have been called the nutrition transition (Popkin, 1994).

Several studies have investigated changes in dietary pattern in Western countries including Latin America and Europe. A relatively similar trends in dietary patterns were reported including an increase in consumption of high-calorie diet, with foods high in saturated fat (mainly from animal sources), added sugars and salt as well as a decrease in using traditional diet, with low intake of complex carbohydrates, dietary fiber, fruits and vegetables (Bermudez and Tucker, 2003; Noah and Truswell, 2003; Mazzocchi et al., 2008).

Similar changes in dietary patterns into Westernized diet have also been reported from developing countries. Rapid industrialization, economic development, and urbanization have resulted in considerable changes in lifestyle and dietary patterns in countries of Asia and Pacific region including Iran (Vorster et al., 1999; Powles, 1992).

Further investigations have also shown a strong correlation between nutrition transition and the prevalence of chronic diseases, especially cancers, suggesting dietary factors as important modifiable determinants for different types of cancers including esophageal cancer (Zhao et al., 2014), breast cancer (Mobarakeh et al., 2014), prostate cancer (Askari et al., 2014) and colorectal cancer (Arafa 
et al., 2011; Yusof et al., 2012).

Golestan province located in Northern Iran has been known as high-risk area for upper gastrointestinal cancers since the 1970s (Mahboubi et al., 1973). The results of recent studies form this region showed increasing trends in the incidence of colorectal and breast cancers (Roshandel et al., 2012).

Regarding the importance of nutrition transition on cancers, as well as high incidence of cancers in this region, we conducted this study to assess the trends of dietary changes in Golestan province between 2001 and 2010.

\section{Materials and Methods}

Source of data: Data on household food consumption was obtained from the household income and expenditure survey (HIES) during 2001 and 2010. The HIES was run by the statistical center of Iran (SCI) throughout all provinces of Iran (Population and census office, 2010). Data was collected at household levels. In other words, each household was considered as a study unit. A threestage sampling was used for selecting eligible samples (households). The first, second and the third stages were areas (cities), clusters and households, respectively. Each cluster included the villages (in rural areas) or blocks (in urban areas). Based on the total number of households in each province, the appropriate sample size was allocated to the areas and clusters. Data was collected by expert and trained interviewers using a structured questionnaire. The questionnaire of HIES was designed considering the recommendations of the United Nations (UN) and according to the national household survey capability programme (NHSCP) (United Nations, 1989) and system of national accounts (SNA) publications (Inter-Secretariat Working Group on National Accounts, 1993). The questionnaire consisted on two major sections including income and expenditure. The section of expenditure was sub-classified into dietary and non-dietary parts.

The dietary part of HIES questionnaire consisted on a list of different food groups including cereals (bread, flour, noodles and its products), meats (red meat, processed meat, poultry meat), fish (fish, shrimp, its products), dairy products (milk, cheese, butter, cream, crud), oils (vegetable oil, animal fat, ghee), fruits (tree

Table 1. Numbers (N) and Proportions (\%) of Households Recruited from Urban and Rural Areas of Golestan Province, Iran

\begin{tabular}{ccccrr}
\hline Year & \multicolumn{2}{c}{ Urban } & \multicolumn{2}{c}{ Rural } & Total \\
& $\mathrm{N}$ & $\%$ & $\mathrm{~N}$ & $\%$ & $\mathrm{~N}$ \\
\hline 2001 & 280 & $37.10 \%$ & 475 & $62.90 \%$ & 755 \\
2002 & 730 & $48.50 \%$ & 775 & $51.50 \%$ & 1505 \\
2003 & 460 & $44.20 \%$ & 580 & $55.80 \%$ & 1040 \\
2004 & 530 & $49.10 \%$ & 550 & $50.90 \%$ & 1080 \\
2005 & 530 & $49.10 \%$ & 550 & $50.90 \%$ & 1080 \\
2006 & 550 & $48.70 \%$ & 580 & $51.30 \%$ & 1130 \\
2007 & 560 & $44.40 \%$ & 700 & $55.60 \%$ & 1260 \\
2008 & 690 & $50.20 \%$ & 685 & $49.80 \%$ & 1375 \\
2009 & 730 & $50.90 \%$ & 705 & $49.10 \%$ & 1435 \\
2010 & 710 & $50.70 \%$ & 690 & $49.30 \%$ & 1400 \\
\hline Total & 5770 & $47.80 \%$ & 6290 & $52.20 \%$ & 12060 \\
\hline
\end{tabular}

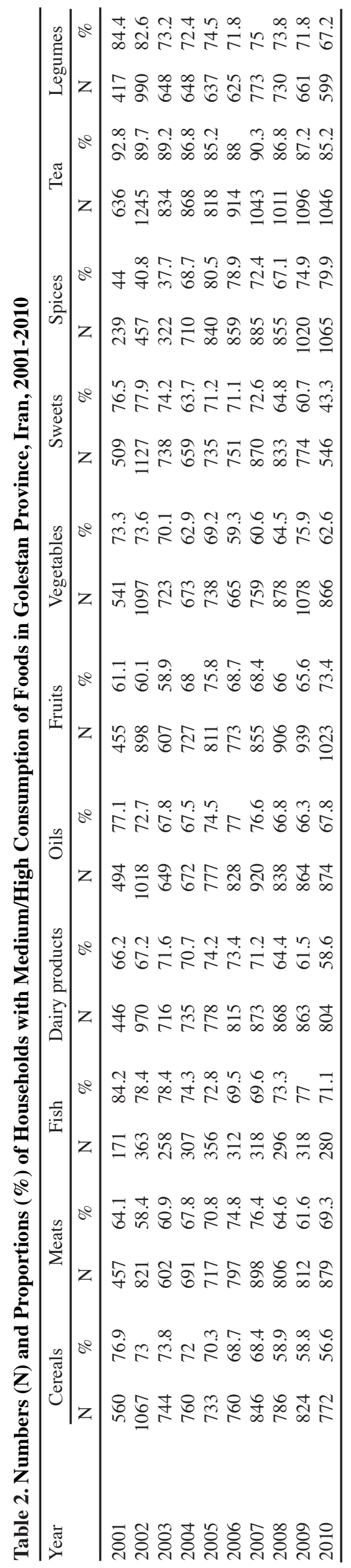


fruits, citrus fruits, berries, melons and nuts), vegetables (leafy vegetables, plant vegetables, root vegetables), sweets (sugar and Jams), spices (condiments and other food composition), tea and legumes.

The questionnaire was filled in by interviewers by referring to selected houses. The amounts of foods (in gram) consumed by the household during the last month was asked and recorded. Month of interview (in each year) as well as the structure of the questionnaire did change during 2001 and 2010.

For the present study we obtained data on dietary part of HIES for Golestan province during 2001-2010 from the SCI.

Statistical analysis: The amounts of food consumption by households were calculated for each of 11 main food groups (cereals, meats, fish, dairy products, oils, fruits, vegetables, sweet, spices, tea and legumes) in each year. Using the tertile distribution of food consumption, households were divided into three categories of food consumption including the 1st tertile (low consumption), the $2^{\text {nd }}$ tertile (medium consumption), and the 3 rd tertile (high consumption). For each year, the proportions of households in each of the tertiles were calculated. Then, the proportions in $2^{\text {nd }}$ or $3^{\text {rd }}$ tertiles were merged into a new group called medium/high consumption. The proportions of households with medium/high consumption of foods were used in final analysis. The aim was to assess temporal variations of the proportions of households with medium/ high consumption of foods between 2001 and 2010 .

We used a Joint point software version 4.0.4 (Statistical Research and Applications Branch, 2013) for trend analysis. Annual percent changes (APCs) in proportions were calculated by generalized linear models assuming a Poisson distribution. Considering the year as independent variable and the neutral logarithm of proportions as dependent variable, a weighted least-square regression was performed. Heteroscedastic errors were used for calculating $95 \%$ confidence intervals $(95 \% \mathrm{CI})$ of APCs. The trend was considered as significant if the $95 \% \mathrm{CI}$ of APC did not include zero. We used previously described pairwise comparison models (Kim et al., 2004) to assess the differences in trends of food consumption between rural and urban households. A P-value of less than 0.05 was considered as significant.

\section{Results}

In total, 12060 households were recruited during 2001 and 2010. Tables 1 shows the number and proportion of households recruited from urban and rural areas. The number and proportion of Golestan households with medium/high consumption of main foods are shown in Table 2. We found significant decreasing trends in the proportions of households with medium/high consumption of vegetables, cereals and legumes during 2001-2010 (Table 3, Figure 1).

The proportions of households with medium/high consumption of sweets and oils showed significant decreasing trends during the study period (Table 3, Figure 2). Figure 3 and Table 3 show that the proportions of households with medium/high consumption of fish and dairy products significantly decreased and those of meat consumption showed significant increasing trend between 2001 and 2010. We found no significant change in the proportions of households with medium/high consumption of spices and fruits (Table 3 ). The proportion of households with medium/high consumption of tea showed a significant decreasing trend in rural area, but the trend was not significant in urban households (Table 3 ).

The results of pairwise comparison analysis showed

Table 3. Annual Percent Change (APC) and 95\% Confidence Interval (95\% CI) of the Proportions of Households with Medium/High Consumption of Foods in Urban and Rural Areas of Golestan Province, Iran, 2001-2010

\begin{tabular}{|c|c|c|c|c|c|c|c|c|c|}
\hline \multirow{3}{*}{$\begin{array}{l}\text { Food group } \\
\text { Cereals }\end{array}$} & \multicolumn{3}{|c|}{ Urban } & \multicolumn{3}{|c|}{ Rural } & \multicolumn{3}{|c|}{ Total } \\
\hline & \multirow{2}{*}{$\frac{\overline{\mathrm{APC}}}{-3.6^{*}}$} & \multicolumn{2}{|c|}{$95 \% \mathrm{CI}$} & \multirow{2}{*}{$\frac{\mathrm{APC}}{-2.5^{*}}$} & \multicolumn{2}{|c|}{$95 \% \mathrm{CI}$} & \multirow{2}{*}{$\frac{\mathrm{APC}}{-3.1^{*}}$} & \multicolumn{2}{|c|}{$95 \% \mathrm{CI}$} \\
\hline & & -4.7 & -2.6 & & -3.4 & -1.5 & & -4.1 & -2.2 \\
\hline Meats & $1.9^{*}$ & 0.2 & 3.6 & $3.4 *$ & 1.8 & 5 & $2.7 *$ & 1.2 & 4.3 \\
\hline Fish & $-2.5^{*}$ & -3.2 & -1.8 & $-3.1^{*}$ & -4 & -2.3 & $-2.8^{*}$ & -3.3 & -2.4 \\
\hline Dairy Products & $-1.0^{*}$ & -1.8 & -0.2 & $-3.0^{*}$ & -4.6 & -1.3 & $-1.9 *$ & -3 & -0.9 \\
\hline Oils & -0.9 & -1.8 & 0 & $-1.3^{*}$ & -2.2 & -0.5 & $-1.2^{*}$ & -2.1 & -0.4 \\
\hline Fruits & 0.5 & -0.4 & 1.5 & 1.3 & -0.1 & 2.8 & 1 & -0.2 & 2.3 \\
\hline vegetables & $-2.5^{*}$ & -3.2 & -1.8 & $-3.5^{*}$ & -4.9 & -2.1 & $-2.9^{*}$ & -3.8 & -2.1 \\
\hline Sweets & $-3.9 *$ & -6 & -1.7 & $-3.5^{*}$ & -5.6 & -1.2 & $-3.7 *$ & -5.6 & -1.7 \\
\hline Spices & 3.9 & -0.5 & 8.4 & 3.5 & -1.9 & 9.3 & 3.8 & -1.1 & 8.9 \\
\hline Tea & -0.1 & -0.8 & 0.5 & $-1.0^{*}$ & -1.6 & -0.5 & $-0.6^{*}$ & -1.1 & 0 \\
\hline Legumes & $-1.8^{*}$ & -2.7 & -0.9 & $-2.7 *$ & -3.8 & -1.6 & $-2.3 *$ & -3.2 & -1.4 \\
\hline
\end{tabular}



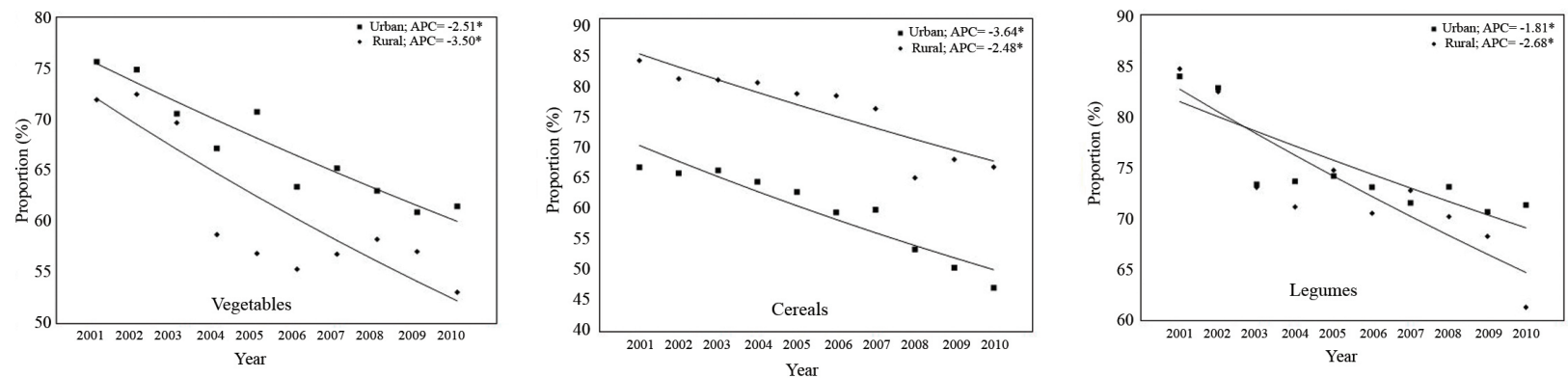

Figure 1. Proportions (\%) of Households with Medium/High Consumption of Vagetables, Cereals and Legumes in Urban and Rural Areas of Golestan Province, Iran, 2001-2010. (APC indicates annual percent change; * indicates that APC is significantly different from zero at alpha $=0.05$; Dots indicate observed values; Solid lines indicate fitted lines)
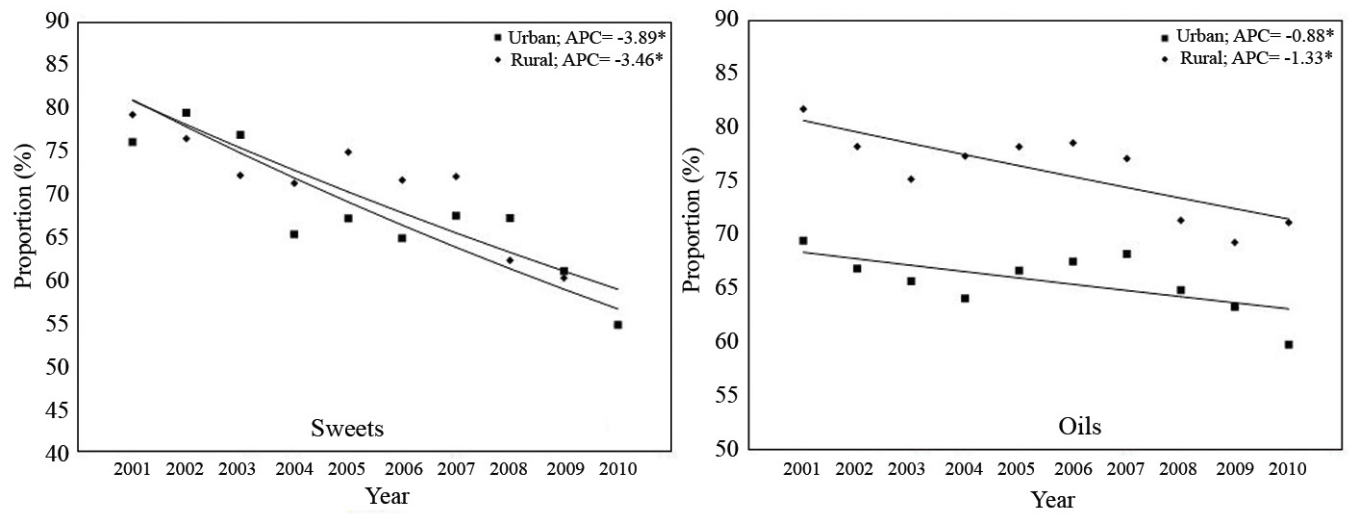

Figure 2. Proportions (\%) of Households with Medium/High Consumption of Sweets and Oils in Urban and Rural Areas of Golestan Province, Iran, 2001-2010. (APC indicates annual percent change; * indicates that APC is significantly different from zero at alpha $=0.05$; Dots indicate observed values; Solid lines indicate fitted lines)
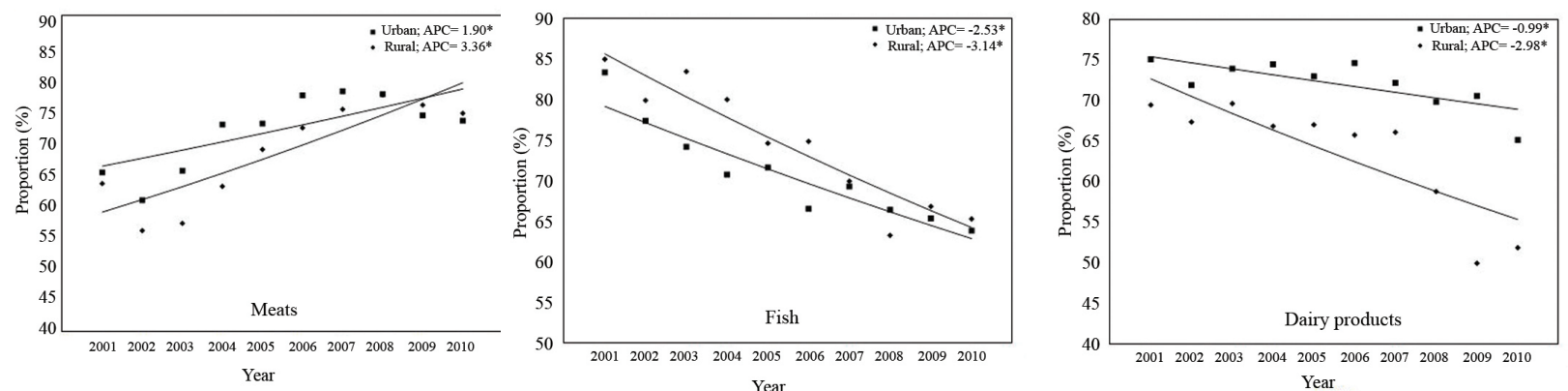

Figure 3. Proportions (\%) of Households with Medium/High Consumption of Meats, Fish and Dairy Products in Urban and Rural Areas of Golestan Province, Iran, 2001-2010. (APC indicates annual percent change; * indicates that APC is significantly different from zero at alpha $=0.05$; Dots indicate observed values; Solid lines indicate fitted lines)

that proportions of households with medium/high consumption of meats $(p=0.01)$, fruits $(p<0.001)$, vegetables $(\mathrm{p}=0.001)$, dairy products $(\mathrm{p}=0.008)$ and tea $(\mathrm{p}=0.009)$ were significantly higher in urban than rural areas. Inversely, the proportions of households with medium/high consumption of fish $(\mathrm{p}=0.008)$, cereals $(\mathrm{p}<0.001)$ and oils $(\mathrm{p}<0.001)$ were significantly lower in urban than rural areas.

\section{Discussion}

Dietary habit is a major indicator of human health. Changes in patterns of food consumption may result in developing different kinds of disease including malignancies. Such changes had been occurred in developed countries in previous decades followed by marked increases in prevalence of none communicable diseases. Developing countries are now prone to experience these changes, called westernization. Therefore, monitoring these trends and, if necessary, implementation of appropriate modifying interventions are important issues in health policy making, especially in cancer control programs, in developing countries. We aimed to assess the variations in patterns of food consumption in Golestan province, a high-risk area for gastrointestinal cancers in Northern Iran.

We found a significant decreasing trend in consumption of plant foods including cereals, vegetables, and legumes as well as fish and dairy products during the last decade.

Lack of adequate knowledge about the benefits of these foods may partly explain such decreasing trends. These foods are usually considered as healthy food, because they meet at least three out of four criteria of healthy food including, 1- to be a good or excellent source of fiber, vitamins, and minerals; 2 - to be high in phytonutrients and antioxidant compounds; 3 - to be low in calorie 
density; and 4- to be readily available (Mayoclinic). These foods have beneficial effects on different organs and may help to reduce the risk of cardiovascular diseases and other health conditions. Low consumption of these foods may cause various conditions including colorectal cancer (Yusof et al., 2012), breast cancer (Mobarakeh et al., 2014), cardiovascular diseases (Kris-Etherton et al., 2003) and osteoporosis (Prentice, 2004). Therefore, educational programs and appropriate interventions should be considered in health policy making to increase consumption of these foods.

Lack of adequate access to these foods may also be considered as another explanation for the observed decreasing trend. Further investigations are warranted to assess different aspects of the decreasing trend in the consumption of these healthy foods in different populations.

According to our results, oils and sweets consumption significantly decreased during the study period. This declining trend may mostly be related to recent policies and interventions (e.g. educational programs) in Iran. Regarding the known effects of these foods on cardiovascular diseases and diabetes (Howard et al., 2006; Weeratunga et al., 2014), a number of programs and interventions were designed and implemented in health system and other related organization of Iran to reduce consumption of these kinds of foods. Our results may indicate that such programs have been effective and their aims have successfully been achieved, suggesting that these programs should be continued and even be reinforced.

We found an increasing trend in the consumption of meats during the last decade. Different studies have shown that high consumption of meats may be related to development of different types of conditions including colorectal cancer (Goldbohm et al., 1994; Arafa et al., 2011; Yusof et al., 2012), cardiovascular diseases (Erlinger and Appel, 2003), metabolic syndrome (Azadbakht and Esmaillzadeh, 2009) and type 2 diabetes (Song et al., 2004). Regarding the increasing trends of malignancies in our region, especially colorectal and breast cancers (Roshandel et al., 2012), it is necessary to design and implement appropriate plans for reducing the consumption of this foods. Therefore, it is recommended to consider this point as a priority in health policy making in this region and other similar populations.

According to our results, consumption of the most of foodstuffs including vegetables, legumes, cereals, fish and dairy products showed decreasing trends during the last decades . These decreasing trends may partly be explained by lack of enough knowledge about beneficences of these of foods. Changes in the sense of taste in our population as well as changes in the socioeconomic status may also explain the decreasing trend in consumption of foodstuffs.

We found differences in foods consumption between residents of rural and urban areas. This may partly be explained by differences in the lifestyle and the levels of socioeconomic status between the two areas. Such space variations in food consumption should be taken into account for conducting dietary modification programs and interventions. Further studies are warranted to
Temporal Variations of Dietary Patterns in Northern Iran assess different aspects of these space disparities in our population and other similar areas.

We found a significant decreasing trend in consumption of healthy foods including plant foods, fish, and dairy products during the last decade. Our results also showed that meats consumption significantly increased during the study period. Low consumption of healthy foods and high consumption of meats may result in developing various conditions, especially cancers (Woo et al., 2014). Therefore, modification in dietary patterns towards higher consumption of healthy foods should be considered as a priority in health policy making in our region as well as other similar population, especially in high-risk populations.

\section{Acknowledgements}

This work was supported by Golestan Research Center of Gastroenterology and Hepatology, Golestan University of Medical Sciences. Authors would like to thank the staff of Statistical center of Iran for providing us the data of the household income and expenditure survey (HIES).

\section{References}

Arafa MA, Waly MI, Jriesat S, et al (2011). Dietary and lifestyle characteristics of colorectal cancer in Jordan: a case-control study. Asian Pac J Cancer Prev, 12, 1931-6.

Askari F, Parizi MK, Jessri M, et al (2014). Dietary patterns in relation to prostate cancer in Iranian men: a case-control study. Asian Pac J Cancer Prev, 15, 2159-63.

Azadbakht L, Esmaillzadeh A (2009). Red meat intake is associated with metabolic syndrome and the plasma C-reactive protein concentration in women. $J$ Nutr, 139, 335-9.

Bermudez OI ,Tucker KL (2003). Trends in dietary patterns of Latin American populations. Cad Saude Publ, 19, 87-99.

Drewnowski A , Popkin BM (1997). The nutrition transition: new trends in the global diet. Nutr Rev, 55, 31-43.

Erlinger T, Appel L (2003). The Relationship between meat intake and cardiovascular disease. Baltimore: Johns Hopkins Center for a Livable Future.

Goldbohm RA, van den Brandt PA, van 't Veer P, et al (1994). A prospective cohort study on the relation between meat consumption and the risk of colon cancer. Cancer Res, $\mathbf{5 4}$, 718-23.

Howard BV, Van Horn L, Hsia J, et al (2006). Low-fat dietary pattern and risk of cardiovascular disease: the women's health initiative randomized controlled dietary modification Trial. JAMA, 295, 655-66.

Inter-Secretariat Working Group on National Accounts (1993). System of national accounts, New York, United Nations.

Kim HJ, Fay MP, Yu B, et al (2004). Comparability of segmented line regression models. Biometrics, 60, 1005-14.

Kris-Etherton PM, Harris WS, Appel LJ (2003). Fish consumption, fish oil, omega-3 fatty acids, and cardiovascular disease. Arterioscler Thromb Vasc Biol, 23, 20-30.

Mahboubi E, Kmet J, Cook PJ, et al (1973). Oesophageal cancer studies in the caspian littoral of Iran: the caspian cancer registry. Br J Cancer, 28, 197-214.

Mayoclinic. 10 great health foods [Online]. Myo Foundation for Medical Education and Research Available: http://www. mayoclinic.org/healthy-living/nutrition-and-healthy-eating/ multimedia/health-foods/sls-20076653 [Accessed 14 May 2014. 


\section{Faezeh Salamat et al}

Mazzocchi M, Brasili C, Sandri E (2008). Trends in dietary patterns and compliance with world health organization recommendations: a cross-country analysis. Public Health Nutr, 11, 535-540.

Mobarakeh ZS, Mirzaei K, Hatmi N, et al (2014). Dietary habits contributing to breast cancer risk among Iranian women. Asian Pac J Cancer Prev, 15, 9543-7.

Noah A ,Truswell S (2003). Commodities consumed in Italy, Greece and other Mediterranean countries compared with Australia in 1960s and 1990s. Asia Pac J Clin Nutr, 12, 23-29.

Popkin BM (1994). The nutrition transition in low-income countries: an emerging crisis. Nutr Rev, 52, 285-98.

Population and census office (2010). Household income and expenditure survey, tehran, statistical center of Iran.

Powles J (1992). Changing lifestyles and health background paper for technical discussions at $43^{\text {rd }}$ session of Regional Committee, September, 1991, World Health Organization, Regional Office for the Western Pacific, Manila, Philippines. Asia Pac J Clin Nutr, 1, 113-26.

Prentice A (2004). Diet, nutrition and the prevention of osteoporosis. Public Health Nutr, 7, 227-243.

Roshandel G, Sadjadi A, Aarabi M, et al (2012). Cancer incidence in golestan province: report of an ongoing population-based cancer registry in Iran between 2004 and 2008. Arch Iran Med, 15, 196-200.

Song Y, Manson JE, Buring JE, et al (2004). A prospective study of red meat consumption and type 2 diabetes in middle-aged and elderly women: the women's health study. Diabetes Care, 27, 2108-15.

Statistical Research and Applications Branch (2013). Joinpoint regression program, version 4.0.4. Bethesda: National Cancer Institute.

United Nations (1989). National household survey capability programme, household income and expenditure surveys: a technical study, New York, United Nations.

Vorster HH, Bourne LT, Venter CS, et al (1999). Contribution of nutrition to the health transition in developing countries: a framework for research and intervention. Nutr Rev, 57, 341-349.

Weeratunga P, Jayasinghe S, Perera Y, et al (2014). Per capita sugar consumption and prevalence of diabetes mellitusglobal and regional associations. BMC Public Health, 14, 186.

Woo HD, Park S, Oh K, et al (2014). Diet and cancer risk in the Korean population: a meta- analysis. Asian Pac J Cancer Prev, 15, 8509-19.

World Health Organization (2003). Diet, nutrition and the prevention of chronic diseases. 2003, Joint WHO/FAO expert consultation. WHO technical report series no. 916, Geneva, WHO.

Yusof AS, Isa ZM, Shah SA (2012). Dietary patterns and risk of colorectal cancer: a systematic review of cohort studies (2000-2011). Asian Pac J Cancer Prev, 13, 4713-7.

Zhao L, Liu CL, Song QK, et al (2014). Association between dietary behavior and esophageal squamous cell carcinoma in Yanting. Asian Pac J Cancer Prev, 15, 8657-60. 